\title{
The Method of Joint Compensation of Reactive Power, Load Balancing and Filtering Harmonics Currents of an Asymmetric Nonlinear Load Connected in a Triangle
}

\author{
Mikhail A. Sherkunkov and Stepan G. Tiguntsev* \\ Irkutsk National Research Technical University \\ Irkutsk, Russian Federation
}

Received 05.03.2020, received in revised form 25.03.2020, accepted 09.04.2020

Abstract. This article explores the method of joint suppression of harmonic currents, balancing currents and partial compensation of the reactive power of a non-linear asymmetric load connected in a triangle using a device installed on the low voltage side of a power transformer.

Keywords: electric energy quality, asymmetry, harmonics, reactive power compensation, modeling, reverse sequence currents.

Citation: Sherkunkov M.A., Tiguntsev S.G. The method of joint compensation of reactive power, load balancing and filtering harmonics currents of an asymmetric nonlinear load connected in a triangle, J. Sib. Fed. Univ. Eng. \& Technol., 2020, 13(4), 455-461. DOI: 10.17516/1999-494X-0237

(C) Siberian Federal University. All rights reserved

This work is licensed under a Creative Commons Attribution-Non Commercial 4.0 International License (CC BY-NC 4.0).

* Corresponding author E-mail address: sherkunkov@mail.ru, stiguncev@yandex.ru 


\title{
Метод совместной компенсации реактивной мощности, симметрирования нагрузки и фильтрации токов \\ гармоник несимметричной нелинейной нагрузки, соединенной в треугольник
}

\author{
М.А. Шеркунков, С.Г. Тигунцев \\ Иркутский национальный исследовательский \\ технический университет \\ Российская Федерация, Иркутск
}

\begin{abstract}
Аннотация. В данной статье исследуется метод совместного подавления токов гармоник, симметрирования токов и частичной компенсации реактивной мощности нелинейной несимметричной нагрузки, соединенной в треугольник, с помощью устройства, устанавливаемого на стороне низкого напряжения силового трансформатора.

Ключевые слова: качество электрической энергии, несимметрия, гармоники, компенсация реактивной мощности, моделирование, токи обратной последовательности.
\end{abstract}

Цитирование: Шеркунков, М.А. Метод совместной компенсации реактивной мощности, симметрирования нагрузки и фильтрации токов гармоник несимметричной нелинейной нагрузки, соединенной в треугольник / М.А. Шеркунков, С.Г. Тигунцев // Журн. Сиб. федер. ун-та. Техника и технологии, 2020. 13(4). С. 455-461. DOI: 10.17516/1999-494X-0237

\section{Введение}

Мощные несимметричные нелинейные нагрузки, подключенные к сети 6-35 кВ, ухудшают качество электрической энергии (КЭЭ) в сети, питающей данную нагрузку. Примерами такой нагрузки являются дуговые сталеплавильные печи, железнодорожные электровозы и т.п. Ухудшение КЭЭ приводит к ухудшению условий работы других потребителей, например к дополнительному нагреву и сокращению срока службы электрооборудования [1], к увеличению потерь активной мощности в элементах сети [2], к ложному срабатыванию релейной защиты. Для улучшения КЭЭ используют специальные устройства, которые компенсируют реактивную мощность, несимметрию токов и токи гармоник нагрузок.

\section{Анализ существующих решений}

Существующие устройства улучшения показателей качества электрической энергии выполняют одну функцию - фильтрацию токов гармоник, симметрирования нагрузки, компенсации реактивной мощности, или совмещают в себе две функции, например, симметрирования и компенсации реактивной мощности нагрузки [3], фильтрации токов гармоник и компенсации реактивной мощности нагрузки [4].

В некоторых случаях необходимо единое устройство, способное совмещать в себе фильтрацию токов гармоник, симметрирование токов нагрузки и компенсацию реактивной мощности.

Целью данного исследования является разработка математической модели и экспериментальная проверка работы на модели устройства, осуществляющего совместную фильтрацию токов гармоник, симметрирование токов нагрузки и компенсацию реактивной мощности.

$$
-456-
$$




\section{Предлагаемое решение}

Для решения проблемы ухудшения КЭЭ нелинейными и несимметричными нагрузками в сетях 6-35 кВ предлагается устанавливать на стороне низкого напряжения силового трансформатора, питающего данную нагрузку, активное фильтро-симметро-компенсирующее устройство (АФСКУ), которое функционально состоит из двухфазного активного фильтра и трехфазного СКУ [5]. Схема трансформатора с нагрузкой и с установленным АФСКУ изображена на рис. 1.

На рис. 1 изображено: 1 - силовой трансформатор; 2, 3, 4 - фазные провода; 5 - несимметричная, нелинейная нагрузка, соединенная в треугольник; 6, 7, 12, 13 - трансформаторы тока; 8, 15 - измерительные блоки; 9, 16 - вычислительные блоки; 10, 17 - формирователи импульсов; 11 - управляемые источники тока активного фильтра; 18 - управляемые источники тока СКУ; 14 - трансформатор напряжения. Алгоритм работы устройства следующий. Трансформаторы тока 6, 7 измеряют токи в фазных проводах «а» и «b», после чего измеренные величины поступают в измерительный блок 8 , где аналоговые значения преобразуются в цифровые. Цифровые значения токов подают в вычислительный блок 9, где рассчитывают разницу между измеренными величинами токов и их первыми гармониками. Эту разность в противофазе подают на формирователь импульсов 10 , который формирует токи в источниках тока активного фильтра 11. Сформированные токи подают в фазные провода 2, 3, 4, в результате чего подавляются токи всех гармоник. Далее измеряют токи в фазных проводах «а» и «b» трансформаторами тока 12, 13. Полученные значения подают в измерительный блок 15 и после

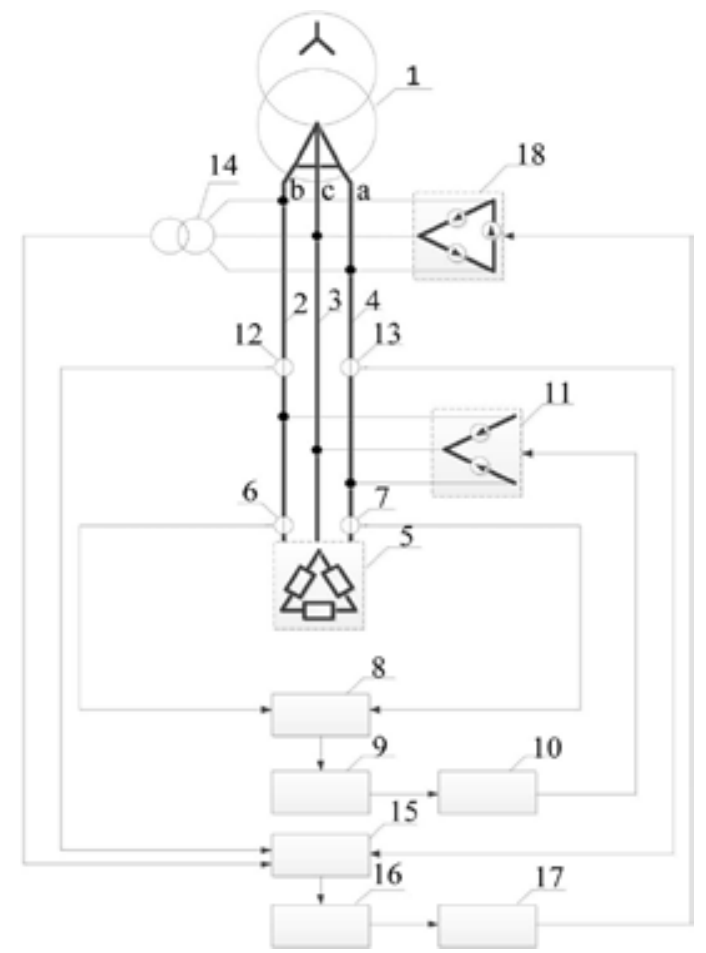

Рис. 1. Схема трансформатора с нагрузкой и с АФСКУ

Fig. 1. Diagram of a transformer with load and with AFSKU 
преобразования в цифровые значения подают в расчетный блок 16, где по формулам (1) и (2) рассчитывают значения токов прямой и обратной последовательностей:

$$
\begin{aligned}
& I_{1}=\frac{a \dot{I}_{b}-a^{2} \dot{I}_{a}}{3}, \\
& I_{2}=\frac{a I_{a}-a^{2} \dot{I}_{b}}{3} .
\end{aligned}
$$

По значениям токов прямой и обратной последовательностей рассчитываются значения сопротивлений СКУ по формулам (3):

$$
\begin{aligned}
& X_{a b}=\frac{-3 U_{1 A}\left(I_{1 p}-\sqrt{3} I_{2 A}+I_{2 p}\right)}{I_{1 A}^{2}+\left(I_{1 p}-\sqrt{3} I_{2 A}+I_{2 p}\right)}, \\
& X_{b c}=\frac{-3 U_{1 A}\left(I_{1 p}-2 I_{2 p}\right)}{I_{1 A}^{2}+\left(I_{1 p}-2 I_{2 p}\right)}, \\
& X_{c a}=\frac{-3 U_{1 A}\left(I_{1 p}+\sqrt{3} I_{2 A}+I_{2 p}\right)}{I_{1 A}^{2}+\left(I_{1 p}+\sqrt{3} I_{2 A}+I_{2 p}\right)} .
\end{aligned}
$$

Где $U_{1 A}$ - значение фазного напряжения прямой последовательности, $\mathrm{B} ; I_{1 A}, I_{1 P}-$ значения активной и реактивной составляющей тока прямой последовательности $I_{1} \mathrm{CКУ.} \mathrm{Обычно} I_{1 A}$ равно нулю, а $I_{1 P}$ определяет величину необходимой компенсации реактивной мощности нагрузки, А; $I_{2 A}, I_{2 P}-$ значения активной и реактивной составляющей тока обратной последовательности $I_{2} \mathrm{CKУ,} \mathrm{которые} \mathrm{определяются} \mathrm{величиной} \mathrm{тока} \mathrm{обратной} \mathrm{последовательности}$ нагрузки, А.

Значения сопротивлений служат для расчета добавочных токов СКУ $\Delta \dot{I}_{a b}, \Delta \dot{I}_{b c}, \Delta \dot{I}_{c a}$ :

$$
\begin{aligned}
& \dot{I}_{a b}=\frac{\dot{U}_{a b}}{j X_{a b}}, \\
& \dot{I}_{b c}=\frac{\dot{U}_{b c}}{j X_{b c}}, \\
& \dot{I}_{c a}=\frac{\dot{U}_{c a}}{j X_{c a}} .
\end{aligned}
$$

По полученным значениям добавочных токов с помощью формирователя импульсов 17 формируются мгновенные синусоидальные значения добавочных токов $\Delta i_{a b}, \Delta i_{b c}, \Delta i_{c a}$, которые подаются в сеть управляемыми источниками тока СКУ 18 в следующий период основной частоты.

\section{Моделирование АФСКУ}

Для исследования режимов работы АФСКУ построена в МАТЛАБе модель трансформатора, питающего нелинейную несимметричную нагрузку, соединенную в треугольник, с установленным АФСКУ. Модель изображена на рис. 2.

\section{Результат}

В результате моделирования получены осциллограммы токов в сети 35 кВ, питающей нелинейную несимметричную нагрузку до включения АФСКУ, после включения АФСКУ и при резком изменении нагрузки. 


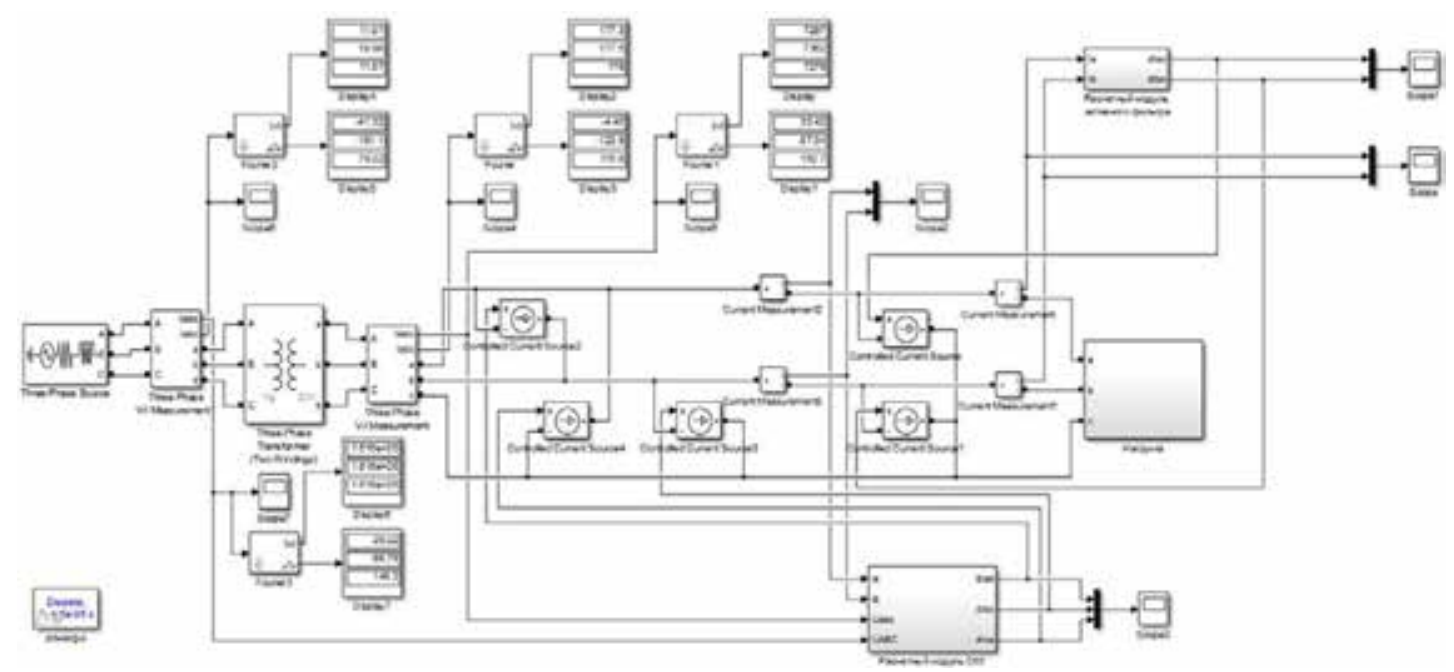

Рис. 2. Модель трансформатора с нагрузкой и с АФСКУ

Fig. 2. Transformer model with load and with AFSKU

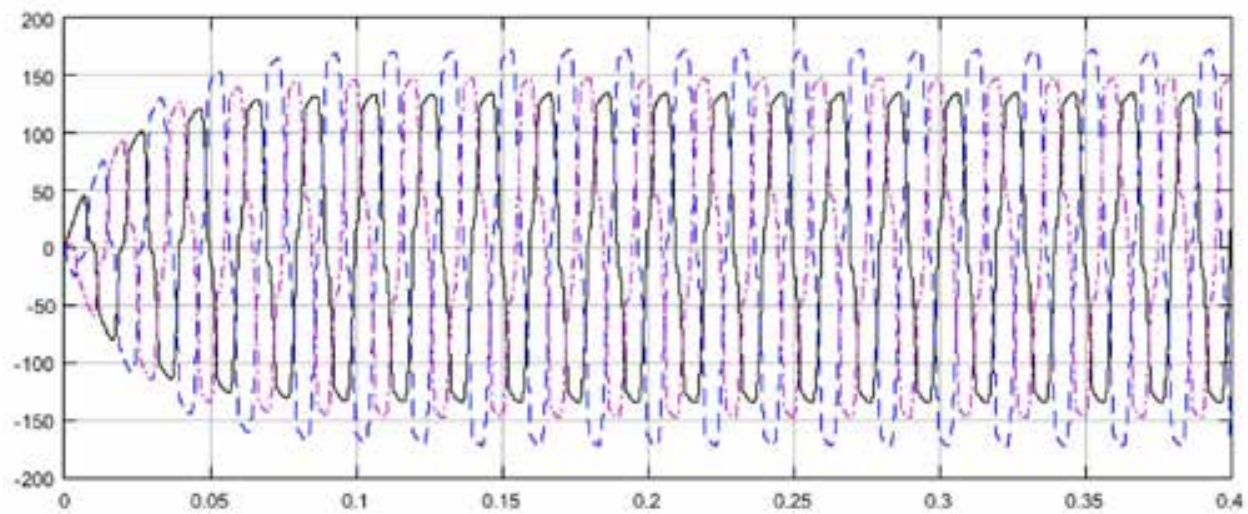

Рис. 3. Осциллограмма токов нагрузки до включения АФСКУ

Fig. 3. Oscillogram of load currents before turning on AFSKU

На рис. 3 изображена осциллограмма токов нагрузки до включения АФСКУ, токи в ампеpax.

Из осциллограммы видно, что токи несинусоидальны и несимметричны. Осциллограмма токов нагрузки после включения АФСКУ изображена на рис. 4.

На осциллограмме видно, что токи в фазных проводах на отрезке времени от 0 до 0,1 ceкунды несинусоидальны и несимметричны. В момент времени 0,1 с включается активный фильтр и происходит подавление токов гармоник, на отрезке времени от 0,1 до 0,2 с токи синусоидальны и несимметричны. В момент времени 0,2 с в работу включается СКУ, происходит подавление токов обратной последовательности нагрузки и полная компенсация реактивной составляющей тока прямой последовательности нагрузки, вследствие чего на отрезке времени от 0,2 с и до конца моделирования токи в фазных проводах синусоидальны и симметричны.

$$
-459-
$$




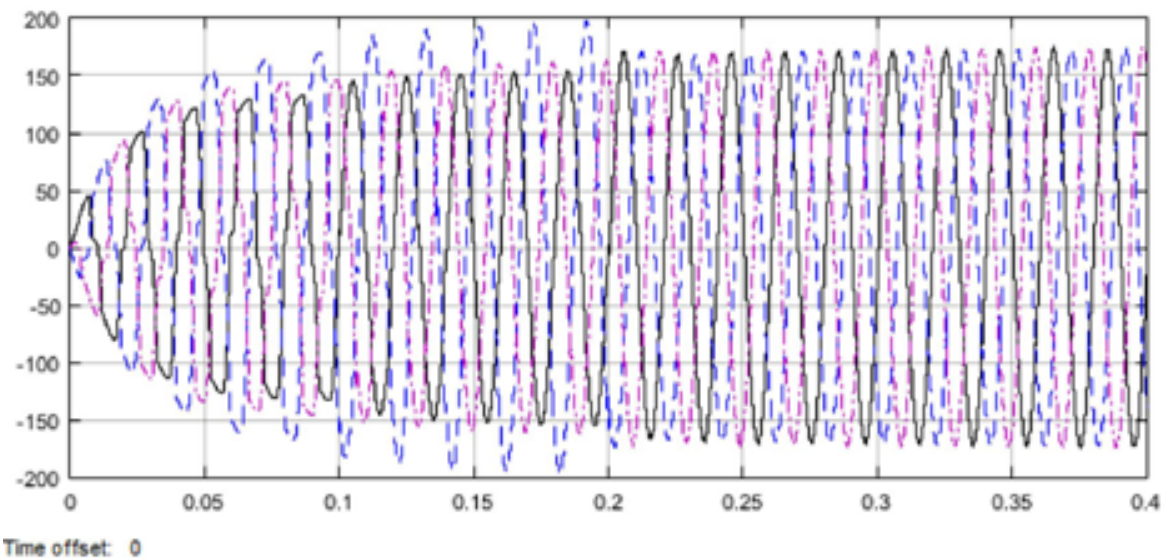

Рис. 4. Осциллограммы токов нагрузки после включения АФСКУ

Fig. 4. Oscillograms of load currents after turning on the AFSKU

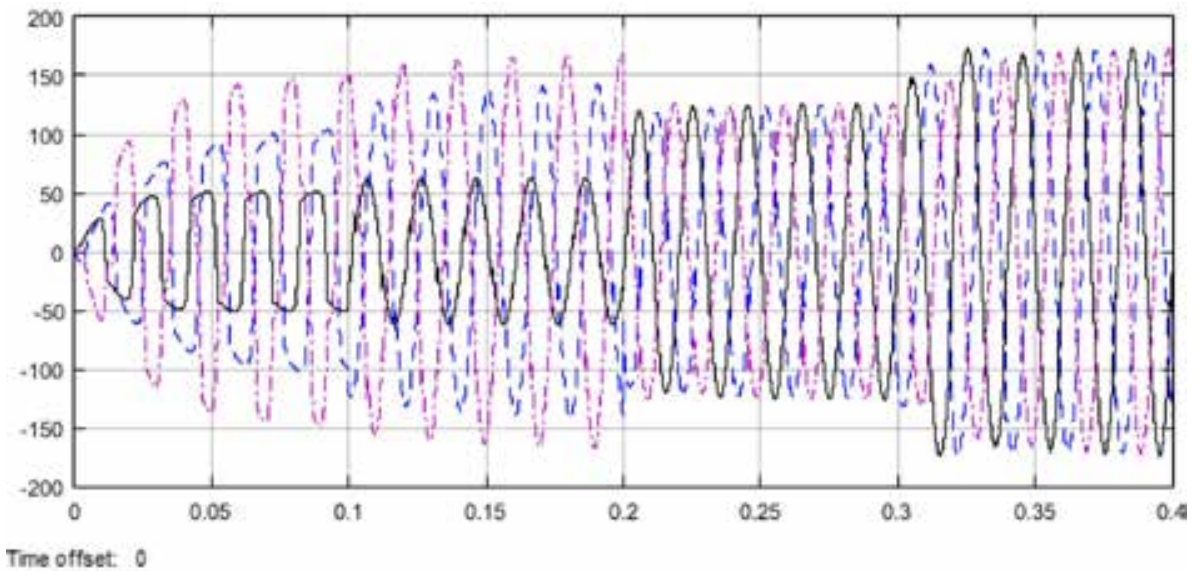

Рис. 5. Осциллограмма токов при изменении мощности нагрузки во время работы АФСКУ

Fig. 5. Oscillograms of load currents after turning on the AFSKU

На рис. 5 изображена осциллограмма токов в фазных проводах при изменении мощности нагрузки при работе АФСКУ.

На осциллограмме видно, что в момент времени 0,3 с произошло резкое изменение мощности нагрузки и начался переходный процесс. В момент времени 0,33 с переходный процесс завершился, токи вновь стали синусоидальны и симметричны.

\section{Выводы}

В результате исследования режимов работы активного фильтро-симметро-компенсирующего устройства выявлено, что АФСКУ позволяет осуществлять подавление токов гармоник, симметрирование токов нагрузки и компенсацию реактивной мощности нагрузки в заданном диапазоне в режиме реального времени. 


\section{Список литературы / References}

[1] Горелов Ю.И., Авдошин В.С. Высшие гармоники в сетях электроснабжения. Известия Тульского государственного университета. Технические науки, 2012, 12(3), 11-16 [Gorelov Y.I., Avdoshin V.S. Higher harmonics in power supply networks. Bulletin of Tula State University. Engineering, 2012, 12 (3), 11-16 (in Russian)]

[2] Черепанов А.В., Тихомиров В.А., Куцый А.П. Влияние несимметрии напряжений на энергоэффективность. Журнал ИрГУПС. Современные технологии. Системный анализ. Моделирование, 2017, 56(4), 207-217 [Cherepanov A.V., Tikhomirov V.A., Kutsyy A.P. The effect of voltage asymmetry on energy efficiency. Magazine IrGUPS Modern technologies. System analysis. Modeling, 2017, 56 (4), 207-217 (in Russian)]

[3] Пат. 2396663 Российская Федерация МПК Н02J 3/12, В60М 3/04 Устройство для симметрирования и повышения коэффициента мощности электротяговой нагрузки/Ефимов А.В., Неугодников А.Ю., Неугодников Ю.П.; заявитель и патентообладатель ОАО «Российские железные дороги». - № 2009121666/09; заявл. 08.06.2009; опубл. 10.08.2010 [Pat. 2396663 Russian Federation IPC H02J 3/12, B60M 3/04 Device for balancing and increasing the power factor of electric traction load / Efimov A.V., Neugodnikov A.Yu., Neugodnikov Yu.P.; Applicant and patent holder of Russian Railways OJSC. - No. 2009121666/09; declared 06/08/2009; publ. 08/10/2010 (in Russian)]

[4] Пат. 2354025 Российская Федерация МПК Н02J 3/18. Способ компенсации высших гармоник и коррекции коэффициента мощности сети /Полищук В.В., Абрамович Б.Н., Сычев Ю.А.; заявитель и патентообладатель Санкт-Петербургский государственный горный институт имени Г.В. Плеханова. - № 2009146366/07; заявл. 14.12.2009; опубл. 27.02.2011 [Pat. 2354025 Russian Federation IPC H02J 3/18. A method for compensating higher harmonics and correcting the network power factor / Polishchuk V.V., Abramovich B.N., Sychev Yu.A.; applicant and patent holder St. Petersburg State Mining Institute named after G.V. Plekhanov. - No. 2009146366/07; declared 12/14/2009; publ. 02/27/2011 (in Russian)]

[5] Пат. 2669770 Российская Федерация МПК Н02J 3/01 Способ совместной частичной компенсации реактивной мощности, подавления токов высших гармоник и симметрирования токов тяговой нагрузки железной дороги / Тигунцев С.Г.; заявитель и патентообладатель Тигунцев С.Г. - № 2017144753; заявл. 19.12.2017; опубл. 16.10.2018 [Pat. 2669770 Russian Federation IPC H02J 3/01 Method for joint partial compensation of reactive power, suppression of higher harmonics currents and balancing of currents of traction load of a railway / Tiguntsev SG; Applicant and patent holder S. Tiguntsev - No. 2017144753; declared 12/19/2017; publ. 10/16/2018 (in Russian)] 